\title{
Acadêmicos, a percepção sobre o papilomavírus humano e sua relação com o câncer cervical
}

\section{Academics, perception about the human papillomavirus and its correlation with cervical cancer}

\author{
Nara Nyely Noronha Luz'; Ítalo Rosal Lustosa²; Kátia da Conceição Machado³; Ana \\ Carolina Landim Pacheco ; Márcia Maria Mendes Marques ; Ana Paula Peron ${ }^{6}$; Paulo \\ Michel Pinheiro Ferreira ${ }^{7}$
}

\begin{abstract}
Resumo
Este trabalho avaliou o conhecimento dos alunos do Curso de Ciências Biológicas da Universidade Federal do Piauí, Campus Senador Helvídio Nunes de Barros sobre o Papilomavírus Humano (HPV). A maioria dos estudantes universitários $(n=218)$ eram do sexo feminino (78\%), com idade entre 17 e 34 anos $(60 \%)$, onde $84 \%$ eram solteiros e com renda familiar entre 2-5 salários mínimos (64\%). A partir dos resultados, verificouse que $87 \%$ conhecem o HPV, $83 \%$ o consideram como uma DST, $68 \%$ acreditam que tanto o homem quanto a mulher pode transmitir e se infectar pelo HPV, $48 \%$ acreditam na possibilidade de transmissão vertical, 35\% consideram que o HPV possa infectar genitálias, cabeça e pescoço, $60 \%$ dos universitários responderam que o exame laboratorial utilizado para a prevenção e detecção inicial da infecção é o Papanicolau. Em relação aos fatores de risco, 64\% afirmaram ter vida sexual ativa, 50\% iniciaram sua vida sexual entre 13 e 17 anos, $44 \%$ usam preservativo durante relações sexuais, $63 \%$ consomem bebidas alcoólicas e $94 \%$ não fumam. A maioria afirmou (64\%) não existir vacina contra o HPV, 52\% acreditam em uma relação entre o HPV e o câncer de colo uterino e 97\% gostariam de ler mais informações sobre o HPV. Assim, verificou-se que os alunos universitários revelaram conhecimento primário sobre o HPV e sobre formas de prevenção da doença. No entanto, quase a metade dos entrevistados desconhece a gravidade da infecção ocasionada por HPV como causa elementar para o surgimento de cânceres de colo uterino.
\end{abstract}

Palavras-chave: Carcinogênese. Colo de útero. Estudantes. Infecções por papilomavirus.

\begin{abstract}
This study evaluated the knowledge of students in Biological Sciences at the Federal University of Piauí, Campus Senador Helvidio Nunes de Barros about the Human Papillomavirus (HPV). The majority of
\end{abstract}

${ }^{1}$ Discente do Curso de Ciências Biológicas, Universidade Federal do Piauí, Rua Cícero Duarte, 905, Bairro Junco, CEP 64607-670, Picos, Piauí, Brasil. E-mail: naranyely@yahoo.com.br.

${ }^{2}$ Mestrando do Programa de Pós-Graduação em Farmacologia, Universidade Federal do Ceará, Bairro Rodolfo Teófilo, CEP 60.430270, Fortaleza, Ceará, Brasil. E-mail: italo.rosal@gmail.com.

${ }^{3}$ Mestranda do Programa de Pós-Graduação em Ciências Farmacêuticas, Universidade Federal do Piauí, Bairro Ininga, CEP 64.049550, Teresina, Piauí, Brasil. E-mail: katiamachado05@hotmail.com.

${ }^{4}$ Docente do Departamento de Ciências Biológicas, Universidade Federal do Piauí, Rua Cícero Duarte, 905, Bairro Junco, CEP 64607670, Picos, Piauí, Brasil. E-mail: carolandim@hotmail.com;

${ }^{5}$ Docente do Departamento de Ciências Biológicas, Universidade Federal do Piauí, Rua Cícero Duarte, 905, Bairro Junco, CEP 64607670, Picos, Piauí, Brasil. E-mail: marciammm2003@gmail.com

${ }^{6}$ Docente do Departamento de Ciências Biológicas / Programa de Pós-Graduação em Genética e Melhoramento, Universidade Federal do Piauí, Rua Cícero Duarte, 905, Bairro Junco, CEP 64607-670, Picos, Piauí, Brasil. E-mail: anpapegenpes@hotmail.com.

${ }^{7}$ Docente do Departamento de Biofísica e Fisiologia / Programa de Pós-Graduação em Ciências Farmacêuticas, Centro de Ciências da Saúde, Universidade Federal do Piaú, CEP 64049-550, Teresina, Piaú, Brasil. E-mail: pmpf@ufpi.edu.br (contato para correspondência). 
the college students ( $\mathrm{n}=218$ ) were female (78\%), between 17 and 34 years-old $(60 \%), 84 \%$ were single and with income between 2-5 times the national minimum wage rate (64\%). Based on these results, it was found that $87 \%$ knew about HPV, $83 \%$ consider it as sexually transmitted disease, $68 \%$ believe that both men and women can transmit and be infected with the HPV, $48 \%$ believed in the possibility of vertical transmission, $35 \%$ consider that HPV can infect genitals, head and neck, $60 \%$ of the students affirmed that the laboratorial exam used to prevent and early detection of this infection is the Pap smear. Regarding risk factors, $64 \%$ have an active sexual life, $50 \%$ started their sexual life between 13 and 17 years of age, $44 \%$ use condoms during sexual intercourse, $63 \%$ consume alcohol, and 94\% do not smoke. The majority stated there is no HPV vaccine (64\%), 52\% believe in the relationship between HPV and cervical cancer and 97\% would like to read more information about HPV. Thus, it was found that academic students revealed primary knowledge about HPV and prevention of disease. However, almost half of the respondents were unaware about the severity of infections caused by HPV as elemental factor to the onset of cervical cancers.

Key words: Carcinogenesis. Cervix. Students. Papillomavirus Infections.

\section{Introdução}

A prática do sexo de forma não responsável acarreta conflitos e pode trazer alterações nos projetos futuros dos jovens, resultando, muitas vezes, em situações de gravidez indesejada, aborto e Doenças Sexualmente Transmissíveis (DSTs) que interferirão grandemente na saúde integral desses indivíduos e no seu futuro social (SCHUSSEL, 2004). A variabilidade de parceiros, o não uso de preservativos e o uso das drogas ilícitas são fatores de risco para o desenvolvimento de doenças sexuais transmissivas (ALTMANN, 2007; CONTI; BORTOLIN; KULKAMP, 2006). Estas doenças apresentam um sério impacto na vida reprodutiva, principalmente, das mulheres em função de poderem causar esterilidade, doenças inflamatórias pélvicas e câncer (BRÊTAS et al., 2009). O Human Papillomavirus (HPV - Papilomavírus humano) se destaca como uma das DST mais comuns no mundo, sendo que uma em cada cinco mulheres é portadora do vírus. A Organização Mundial de Saúde (WHO) estima que cerca de 630 milhões de novos casos em todo o mundo surjam a cada ano (WORLD HEALTH ORGANIZATION, 2013).

A infecção por este vírus tem sido diretamente ligada ao desenvolvimento de cânceres de colo de útero (SANTOS et al., 2003). Estima-se mais de 500 mil novos casos de câncer de colo uterino causado por alguns tipos de HPV por ano, ocasionando a morte de cerca de 270 mil mulheres. Valores superiores a $85 \%$ das mortes causadas por cânceres de colo ocorrem em países em desenvolvimento, sendo responsável por $13 \%$ de todos os cânceres no sexo feminino (WORLD HEALTH ORGANIZATION, 2013).

O Brasil é um dos líderes mundiais em incidência de HPV, onde mais de 130 mil novos casos são registrados a cada ano, na grande maioria em mulheres jovens. Os especialistas chamam a atenção para o crescimento da doença, responsável por $90 \%$ dos casos de câncer de colo de útero. No entanto, sugere-se a ocorrência de 3 a 6 milhões de homens infectados (BRASIL, 2006; QUEIROZ; PESSOA; SOUSA, 2005). Dos mais de 100 tipos de HPV descritos, cerca de 40 são sexualmente transmissíveis. Entretanto, a maioria das infecções causadas pelo HPV é assintomática ou não diagnosticada. As sintomáticas causam verrugas ou papilomas, onde os tipos virais estão associados ao desenvolvimento do câncer do colo do útero (LONGWORTH; LAIMINS, 2004; SCHIFFMAN et al., 1993).

Aproximadamente $40 \%$ das mulheres sexualmente ativas são infectadas pelo HPV (NASCIMENTO et al., 2013) que é transmitido predominantemente pelo contato genital com a pessoa infectada, incluindo sexo oral, por via sanguínea, de mãe para filho no momento do parto (materno-fetal) ou por meio de instrumentos ginecológicos não estéreis. Na maioria das vezes, a infecção é transitória e desaparece sem deixar vestígios, podendo ser sintomática ou assintomática. Por isso, 
quando se realiza o diagnóstico, não se consegue saber se a infecção é recente ou antiga (BRASIL, 2006; SILVA; CRUZ, 2010).

Durante anos, pouca importância foi dada à infecção pelo HPV, uma vez que ela era considerada uma doença benigna. Apenas após o estabelecimento de sua correlação com o câncer de colo uterino, tornouse importante realizar campanhas de prevenção, pois a detecção precoce da infecção permite evitar ou retardar a progressão para o câncer invasivo. A prevenção pode ser dividida em primária, como campanhas de incentivo do uso de preservativos, e secundária, como campanhas de estímulo à realização de exame preventivo (SILVA et al., 2004).

De um modo geral, mudanças ocorridas nas últimas décadas têm alterado o perfil das DSTs, transformando seu controle em um problema de saúde pública, não apenas por sua incidência e prevalência, mas por suas consequências, como as complicações psicossociais e econômicas, pois acometem a grande parcela da sociedade emidade produtiva. Assim, fatores biológicos, falta de informação e conceitos equivocados, facilitam a transmissão de doenças sexuais, com ênfase ao HPV, na adolescência e na juventude reprodutiva. Apesar disso, essa DST ainda não possui um espaço significativo nas campanhas nacionais de educação e prevenção. Portanto, torna-se necessário avaliar o conhecimento e os hábitos sexuais, principalmente dos jovens, como uma forma de averiguar o impacto resolutivo das campanhas educativas, que tem como primazia tornar a população assistida menos susceptível a esse tipo de infecção (TAQUETTE; VILHENA; PAULA, 2004). Assim, este trabalho objetivou avaliar o conhecimento dos alunos do Curso de Ciências Biológicas da Universidade Federal do Piauí sobre o HPV e sua relação com o câncer de útero.

\section{Material e Métodos}

Este estudo é uma análise quantitativa, do tipo descritiva e transversal. Estudos descritivos têm como objetivo principal descrever as características de determinada população ou fatos e fenômenos de determinada realidade. Este tipo de estudo promove um delineamento da realidade já que esta descreve, registra, analisa e interpreta a natureza atual ou o processo dos fatos.

A pesquisa foi realizada na Universidade Federal do Piauí, Campus Senador Helvídio Nunes de Barros, localizada na cidade de Picos, Piaú. Picos é um município com aproximadamente 75 mil habitantes e com grande influência socioeconômica no semiárido nordestino (PICOS, 2008).

$\mathrm{O}$ estudo foi realizado entre os meses de março e novembro de 2013 por meio de entrevista com aplicação de um questionário a 218 alunos pertencentes ao Curso de Licenciatura em Ciências Biológicas da Universidade Federal do Piauí do Campus Senador Helvídio Nunes de Barros (CSHNB/UFPI). O questionário foi composto de quinze perguntas de múltipla escolha a fim de obter principalmente as seguintes informações: sexo, idade, estado civil, renda familiar, início da atividade sexual, uso de preservativos e conhecimento sobre o HPV. Todos os alunos convidados a participar foram esclarecidos quanto ao objetivo da pesquisa. Não houve identificação nominal nem risco moral para os participantes. Os participantes responderam o questionário de forma individual, sem qualquer tipo de consulta e sem interferência do pesquisador.

O Termo de Consentimento Livre Esclarecido (TCLE)foi entregueemduas vias, umapara opesquisador e outra para o participante, como estabelecido pela Resolução 466/2012 do Conselho Nacional de Saúde. Este projeto foi submetido e aprovado pelo Comitê de Ética em Pesquisa da Universidade Federal do Piauí (CAAE-01381812.5.0000.5214). Os resultados foram expressos em valores percentuais utilizando o programa Excel (Windows 2010).

\section{Resultados e Discussão}

Nos últimos anos, observou-se uma verdadeira epidemia com a descoberta das lesões subclínicas causadas por diferentes tipos de HPV. Fatores 
biológicos, falta de informação e conceitos equivocados, facilitam a transmissão de doenças sexuais na adolescência e na juventude. Dentre estas, o HPV não possui ainda um espaço significativo nas campanhas nacionais de educação e prevenção. Por este fato, torna-se necessário avaliar o conhecimento e os hábitos sexuais para que as campanhas educativas alcancem resultados, tornando os jovens menos susceptíveis a esse tipo de infecção (FERNANDES et al., 2000; MURTA et al., 2001; TAQUETTE et al., 2004).

Para a realização deste estudo foram entrevistados 218 estudantes universitários do Curso de Ciências Biológicas, sendo que $78 \%$ foram representados por mulheres e $22 \%$ por homens. A idade dos entrevistados variou entre 17 e 34 anos, sendo que a maioria estava entre 17 e 22 anos (60\%), 37\% entre 23 e 28 anos e $3 \%$ entre 29 e 34 anos de idade. Com relação ao estado civil dos entrevistados, verificouse que $84 \%$ eram solteiros, $12 \%$ casados e $4 \%$ não responderam ao questionamento. Resultados semelhantes foram encontrados por Silveira et al. (2012) em um estudo sobre o conhecimento de universitários sobre HPV de uma faculdade privada localizada no sertão de Pernambuco, revelando que $76,6 \%$ eram solteiros e $23,4 \%$ eram casados.

A maioria dos alunos entrevistados possuía renda familiar entre 2 e 5 salários mínimos (64\%), 34\% possuíam renda até 1 salário mínimo, $1 \%$ renda entre 6 e 10 salários mínimos e $1 \%$ acima de 10 salários mínimos. Oitenta e sete por cento (87\%) dos entrevistados afirmaram conhecer ou que já terem ouvido falar sobre o HPV. Do total de entrevistados, $83 \%$ consideram o HPV como uma DST, 1\% não o consideram como uma DST, 15\% afirmaram não saber e 1\% não responderam. Esse achado é preocupante, uma vez que $15 \%$ dos entrevistados não reconhecem o HPV como um agente etiológico. Um dos fatores responsáveis por tal desconhecimento se deve ao fato de que a disciplina de microbiologia é ministrada apenas no último ano do Curso de Graduação em Ciências Biológicas, mas também sugere a deficiência de conhecimentos básicos adquiridos durante o ensino fundamental e médio e reflete a ausência de campanhas de conscientização sobre as consequências de infecção pelo HPV.

Sessenta e oito por cento (68\%) dos universitários consideraram que tanto o homem quanto a mulher podem transmitir e se infectar pelo vírus do HPV, $25 \%$ responderam não saber e $1 \%$ não responderam. Estimativas mundiais indicam que $20 \%$ dos indivíduos sadios estão contaminados com HPV (LINHARES; VILLA, 2006), dos quais 70\% ocorrem em países em desenvolvimento, sendo as mulheres a maior a parte das vítimas. Porém, os homens, atuando como "portadores" ou "vetores" de tipos oncogênicos, contribuem significativamente para o aumento do risco de ocorrência da transmissão para as parceiras (CASTELLSAGUE et al., 2003).

Quando os alunos foram questionados quanto à possibilidade de transmissão vertical de HPV para o recém-nascido durante o parto, 48\% acreditam nessa possibilidade, 9\% consideram essa possibilidade inexistente e 43\% afirmaram não saber. A gravidez é um fator predisponente à infecção por HPV, devido em parte à diminuição da imunidade celular e à modificação dos hormônios esteroides, fato comprovado clinicamente pela alta taxa de regressão das lesões após o parto (PINTO; TULIO; CRUZ, 2002; YOST et al., 1999). De fato, estudos demonstraram haver uma maior frequência de infecção por HPV em gestantes em relação a não gestantes sugerindo que a gravidez é um fator de risco para infecção pelo HPV. As manifestações clínicas e subclínicas são mais evidentes nas gestantes, sendo que grande parte delas regridem no puerpério. (FIFE et al., 1996).

Cerca de $35 \%$ dos entrevistados consideram que o HPV possa infectar a região genital e a região da cabeça e do pescoço, como boca, laringe e cavidade oral, 14\% não acreditam que essa infecção possa ocorrer e $51 \%$ responderam não saber. O epitélio da cavidade oral e orofaringe é uma membrana mucosa com propensão para o desenvolvimento neoplásico, sendo exposto a vários carcinógenos nutricionais e 
ambientais, determinantes de lesões decorrentes de efeitos da infecção pelo HPV e mutagênese química, sendo que dos 77 tipos de HPV isolados, 41 deles apresentam maior tendência a se instalar nas mucosas (SCULLY, 2002). Na mucosa oral, o HPV está relacionado com a patogênese de lesões verrucosas benignas (papiloma escamoso oral, verruga vulgar, condiloma acuminado e hiperplasia epitelial focal) (FELLER; KHAMMISSA; WOOD, 2009). Embora ainda não seja completamente conhecida, a infecção da mucosa oral pode ocorrer durante a gravidez, no parto vaginal, por inoculação ou sexo oral (CASTRO et al., 2009; MARTINS FILHO et al., 2009).

Quando questionados sobre o exame utilizado para a prevenção e detecção inicial da infecção pelo HPV, verificou-se que $2 \%$ responderam ultrassom, $60 \%$ responderam exame do Papanicolau, 13\% responderam hemograma, $24 \%$ não souberam responder e $1 \%$ não responderam (Figura 1). Cerca de $62 \%$ das universitárias entrevistadas no interior de Pernambuco disseram que reconhecem o exame Papanicolau como procedimento básico no diagnóstico do HPV e o realizam anualmente, embora 24,4\% nunca fizeram o exame, 9,1\% realizou o exame uma única vez e 4,5\% faz o exame a cada 3 anos (SILVEIRA; FERRAZ; CONRADO, 2012).

Figura 1 - Exame utilizado para prevenção e detecção inicial da infecção por HPV segundo a percepção dos entrevistados no Curso de Ciências Biológicas da Universidade Federal do Piauí, Campus Senador Helvídio Nunes de Barros, Picos, Piauí (n=218).

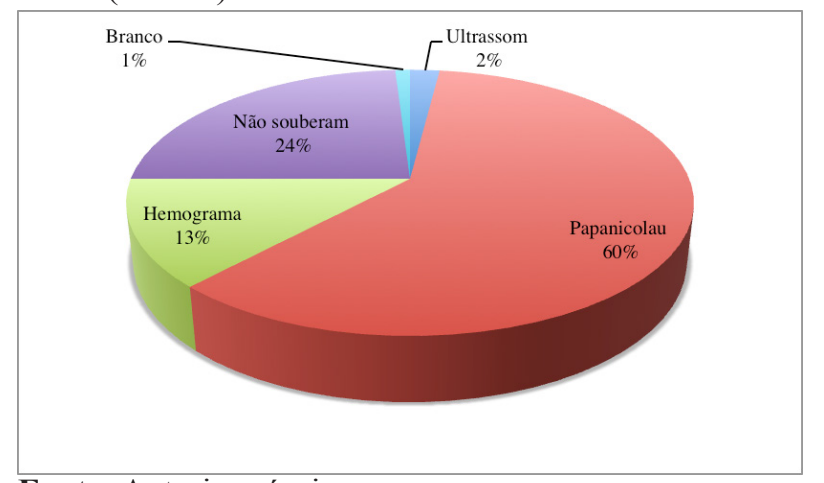

Fonte: Autoria própria.
Os exames de prevenção, com destaque ao exame de Papanicolau, são estratégias importantes para a redução da mortalidade por câncer de colo uterino, vagina e vulva relacionados ao $\mathrm{HPV}$ (QUEIROZ; PESSOA; SOUSA, 2005). No Brasil, a prevenção secundária é realizada através do exame de Papanicolau, um exame sensível, seguro, barato e de especificidade relativamente boa que rastreia lesões cancerígenas em amostras citológicas do colo do útero em suas fases iniciais, antes de elas se tornarem invasivas (FRIGATO; HOGA, 2003; PINHO; FRANÇA-JUNIOR, 2003).

Quando questionados se tinham vida sexual ativa, $64 \%$ dos alunos afirmaram que sim, 35\% afirmaram que não e $1 \%$ não responderam, observando-se que $2 \%$ iniciaram com menos de 12 anos, 50\% entre 13 e 17 anos, $42 \%$ entre 18 e 21 anos e $6 \%$ a partir dos 22 anos de idade. Avaliando o grau de conhecimento de universitários em saúde sobre o HPV, Anticaglia, Souza e Raitz (2008) mostrou que 71,4\% das alunas e $88 \%$ dos alunos afirmaram ser sexualmente ativos. Em outra análise mais recente, o perfil sexual e reprodutivo de 137 estudantes universitários de uma faculdade mostrou que a idade da primeira relação estava na faixa etária acima de 18 anos (44,2\%) e entre 16-18 anos (35,4\%) (SILVEIRA; FERRAZ; CONRADO, 2012).

De acordo com Ramos (2011), estima-se que, a cada ano, quatro milhões de jovens, entre 15 e 17 anos, tornam-se sexualmente ativos no Brasil. Saito (2010) afirma que ocorrem cerca de 12 milhões de transmissões de DST a cada ano, e um terço destas em indivíduos que iniciam sua vida sexual precocemente, possuindo vários parceiros sexuais. Em uma pesquisa realizada por Nascimento et al. (2013), dos 400 adolescentes entrevistados, 337 indivíduos responderam que o HPV se contrai apenas por contato sexual e 336 indivíduos afirmaram que somente a atividade sexual precoce aumenta a probabilidade de se contrair HPV.

Os estudos que avaliaram a relação entre a idade do início da atividade sexual e o risco de infecção 
por HPV consideraram o histórico de atividade do parceiro como grande influência nas frequências de infecção encontradas. Contudo, é necessário ajustar este dado para a idade do início da atividade sexual, sendo estes dois fatores muito importantes em relação ao risco de infecção por HPV (KAHN et al., 2002a). Assim, quanto mais precoce o início das atividades sexuais, maior o risco de infecção (KAHN et al., 2002b).

Na idade de 50 anos, $80 \%$ das mulheres terão sido contaminadas por esse vírus (BROOMALL; REYNOLDS; JOCOBSON, 2010). Entretanto, a infecção é adquirida principalmente na adolescência (SMITH et al., 2008) e as maiores taxas de infecção aparecem nas mulheres sexualmente ativas com até 25 anos de idade (AULT, 2006). Além da idade, outros fatores que aumentam a probabilidade de infecção pelo HPV e desenvolvimento de cânceres de útero em mulheres estão o número elevado de gestações, o uso de contraceptivos orais, tabagismo e outras DSTs (BRASIL, 2006).

$\mathrm{Na}$ vida sexual e reprodutiva, o preservativo masculino é um recurso disponível a homens e mulheres que atende à dupla função de proteção contra a gravidez e contra DSTs, dentre as quais o HPV. Mesmo assim, é comum a resistência ao seu uso tanto homens como por mulheres (MADUREIRA; TRENTINI, 2008).

No que diz respeito ao uso de preservativo nas relações sexuais, verificou-se que $6 \%$ dos entrevistados nunca usaram, $31 \%$ usam somente às vezes, 44\% sempre usam e 19\% não responderam (Figura 2). Como nenhum aluno foi excluído do plano amostral, é provável que os $19 \%$ que não responderam o questionamento sobre uso de preservativo estejam incluídos dentro dos $35 \%$ que afirmaram não possuir vida sexual ativa. No estudo de Silveira, Ferraz e Conrado (2012), a maioria dos universitários $(39,0 \%)$ disseram que usam preservativo somente em algumas relações sexuais, $35,4 \%$ responderam que usam preservativo em todas as relações sexuais e $25,6 \%$ responderam que não usam preservativo. Considerando a utilização do preservativo entre alunos sexualmente ativos, nota-se que nem todos apreciam a importância da prevenção da transmissão de HPV e de outras DSTs e, por isso, nem sempre fazem uso de preservativos, quase sempre alegando que o uso da camisinha diminui o prazer.

Figura 2 - Uso de preservativo em relações sexuais pelos alunos do Curso de Ciências Biológicas da Universidade Federal do Piauí, Campus Senador Helvídio Nunes de Barros, Picos, Piauí (n=218).

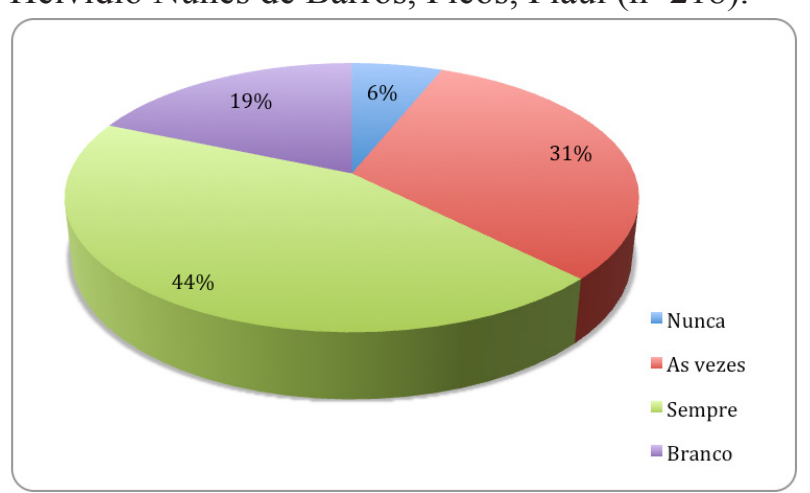

Fonte: Autoria própria.

Taquette, Vilhena e Paula (2004) ressaltam que os adolescentes, em geral, sabem que o preservativo evita doenças e gravidez, mas mesmo assim não o usam. Estes autores ainda reforçam que existe uma enorme lacuna entre o nível de conhecimento e o uso efetivo da "camisinha", onde a juventude aponta numerosas justificativas para não usá-la, como: esquecimento, custo e desprazer na relação sexual. Entretanto, com essa atitude aumentam os riscos de contaminação por DST, como por exemplo, o HPV (ANTICAGLIA; SOUZA; RAITZ, 2008; CONTI; BORTOLIN; KULKAMP, 2006).

Quando questionados se consomem bebida alcoólica, foi verificado que $30 \%$ nunca consumiram, $63 \%$ consomem às vezes e $7 \%$ consomem sempre. Observou-se que $2 \%$ dos alunos são tabagistas assumidos, 94\% afirmaram que não fumam, $3 \%$ afirmaram que fumam às vezes e $1 \%$ não responderam. Os estudos de 
Murta et al. (1997) demonstraram que as mulheres fumantes de diferentes faixas etárias apresentam maior incidência de infecção pelo HPV que as não fumantes. O fumo está relacionado com infecção por HPV e com neoplasias cervicais (ALTMANN, 2007), provavelmente, devido à imunossupressão local causada pelo fumo, que permite a penetração do vírus nas células com maior facilidade (BRÊTAS et al., 2009).

Quando questionados sobre a existência de vacinas contra HPV, 35\% acreditam na existência da vacina, $64 \%$ desconhecem e $1 \%$ não responderam. Duas vacinas contra o HPV já foram aprovadas no Brasil: Cervarix (Glaxo SmithKline ${ }^{\circledR}$ ) e Gardasil (Merck ${ }^{\circledR}$ ). Ambas contêm a proteína L1 do capsídeo viral, produzidas por tecnologia recombinante para a obtenção de partículas análogas às virais (VLPs) dos dois vírus mais comuns nos cânceres cervicais: HPV16 e o HPV18 (HARPER et al., 2006; VILLA et al., 2005).

A utilização da vacina para a prevenção de infecções por HPV ainda é muito controversa, mesmo não havendo riscos de infecção devido a sua administração. Seu uso depende não só da aceitação por médicos, mas pela sociedade, que esbarra em problemas religiosos e morais, que podem ser contornados através de campanhas que informem os riscos associados ao HPV e os benefícios da vacinação (AIRES et al., 2006).

Quando questionados sobre a existência de uma relação direta entre infecção por HPV e câncer de colo de útero, verificou-se que $52 \%$ acreditam que exista essa relação, $4 \%$ não acreditam nessa relação e 44\% afirmaram não saber (Figura 3). Evidências moleculares e epidemiológicas indicam claramente que certos tipos de HPV são a principal causa de câncer cervical. A infecção persistente por um tipo HPV oncogênico é considerada o fator de risco mais importante para o câncer cervical. Enquanto a maioria das pessoas elimina a infecção em até dois anos, muitos tipos possuem alto risco de progressão para câncer (EINSTEIN et al., 2009), sendo o HPV identificado em mais de $99 \%$ dos carcinomas cervicais (BROOMALL; REYNOLDS; JOCOBSON, 2010). Além disso, a infecção está potencialmente associada com 90 a $93 \%$ dos carcinomas anais, 12 a $63 \%$ dos carcinomas da orofaringe, 36 a $40 \%$ dos carcinomas de pênis, 40 a $64 \%$ dos carcinomas vaginais e 40 a $51 \%$ dos carcinomas da vulva (CHATURVEDI, 2010).

Figura 3 - Percepção da existência de uma relação entre infecção por HPV e câncer de colo de útero por alunos do Curso de Ciências Biológicas da Universidade Federal do Piauí, Campus Senador Helvídio Nunes de Barros, Picos, Piauí (n=218).

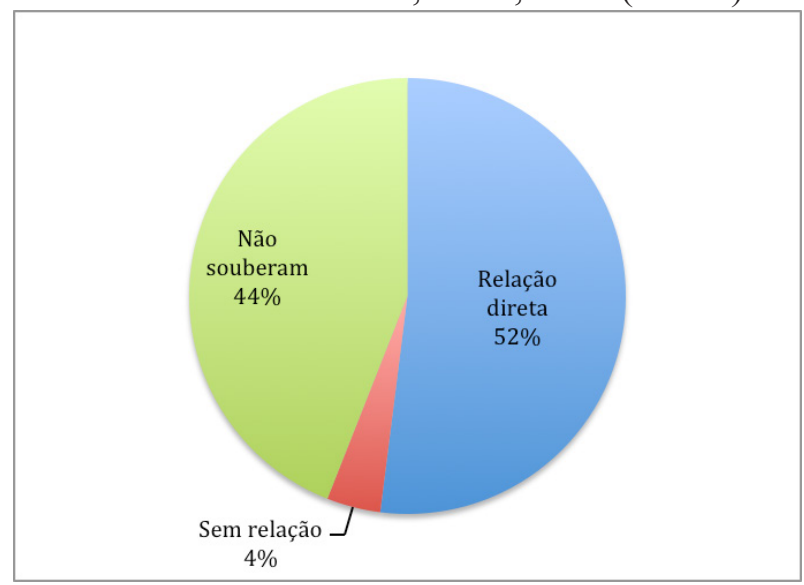

Fonte: Autoria própria.

A síntese do DNA viral ocorre na camada basal do epitélio escamoso, justamente onde surgem as primeiras células neoplásicas. Isto ocorre a partir da síntese de 3 oncoproteínas, sendo que a proteína E5 parece atuar, sinergeticamente, com o fator de crescimento epidérmico (EGF - Epidermal Growth Factor), a E6 liga-se ao p53 selvagem e a E7 liga-se à proteína Retinoblastoma ( $\mathrm{pRb}$ ) e a desativa, todas encontradas em infecções por HPV de alto risco (HPV16, 18, 31, 33, 35, 39 e 42) (CÂMARA et al., 2003).

Quando questionados se tinham interesse em ler uma cartilha educativa com informações sobre o HPV, 97\% responderam que sim e $3 \%$ responderam que não. Reis et al. (2010) 
verificaram que $98 \%$ dos entrevistados aprovaram as cartilhas como material referencial para facilitar a obtenção de informações em saúde, uma vez que elas despertam o interesse pelo assunto em questão.

Assim, é fundamental que os profissionais de saúde sejam capazes de orientar corretamente a população sobre a importância dos exames preventivos. A necessidade de se fazer a detecção e tratamento precoces em lesões pré-malignas causadas por HPV podem perfeitamente prevenir a progressão para o câncer (FEHRMANN; LAIMINS, 2003). Neste contexto, determinar o nível de conhecimento sobre a infecção por HPV e suas consequências em um grupo de estudantes de cursos universitários da área de saúde, futuros profissionais e agentes multiplicadores do conhecimento, possibilita à Universidade um diagnóstico do conhecimento absorvido pelos alunos durante o curso e, consequentemente, um parâmetro que direcione mudanças nas práticas pedagógicas (ANTICAGLIA; SOUZA; RAITZ, 2008).

\section{Conclusão}

Os universitários do Curso de Graduação em Ciências Biológicas revelaram um conhecimento primário sobre o HPV e sobre as formas de prevenção da doença. Nessa mesma linha, quase a metade dos entrevistados desconhece a gravidade da infecção ocasionada por HPV como causa elementar para o surgimento de cânceres de colo uterino. Estas informações servem para nortear ações educativas e reforçar sobre a importância da orientação sexual na escola e na Universidade, e mostrar a necessidade de investimentos no desenvolvimento de práticas de promoção à saúde com atividades educacionais e enfoque adequado a cada faixa etária, usando uma linguagem direta e apropriada, quebrando mitos e desmistificando tabus.

\section{Referências}

AIRES, K. A., CIANCIARULLO, A. M., CARNEIRO, S. M., VILLA, L. L., BOCCARDO, E., PÉREZ-MARTINEZ, G. Production of human papillomavirus type $16 \mathrm{~L} 1$ virus-like particles by recombinant Lactobacillus case cells. Applied and Environmental Microbiology, Washington, v. 72, p. 745-752, 2006.

ALTMANN, H. Educação sexual e primeira relação sexual: entre expectativas e prescrições. Revista Estudos Feministas, Florianópolis, v. 15, p. 333-356, 2007.

ANTICAGLIA, C. M.; SOUZA, P. R. K.; RAITZ, A. Conhecimento de estudantes universitários sobre HPV, sua relação com câncer de útero e métodos preventivos. Revista Brasileira de Ciências da Saúde, São Caetano do Sul, v. 6, n. 15, p. 33-38, 2008.

AULT, K. A. Epidemiology and natural history of human papillomavirus infections in the female genital tract. Infectious Diseases in Obstetrics and Gynecology, Nars City, v. 40, p. 1-5, 2006.

BRASIL. Ministério da Saúde. Manual de controle das doenças sexualmente transmissíveis DST. 4. ed. Brasília: Ministério da Saúde, 2006.

BRÊTAS, J. R. S.; OHARA, C. V. S.; JARDIM, D. P.; MUROYA, R. L. Conhecimentos de adolescentes sobre doenças sexualmente transmissíveis: subsídios para a prevenção. Acta Paulista de Enfermagem, São Paulo, v. 22, p. 786-792, 2009.

BROOMALL, E. M.; REYNOLDS, S. M.; JOCOBSON, R. M. Epidemiology, clinical manifestations, and recent advances in vaccination against human papillomavirus. Postgraduate Medicine, San Diego, v. 122, p. 121-129, 2010.

CÂMARA, G. N. L.; CRUZ, M. R.; VERAS, V. S.; MARTINS, C. R. F. Os papilomavírus humanos HPV: Carcinogênese e Imunogênese. Universitas Ciências da Saúde, Brasília, v. 1, p. 159-168, 2003.

CASTELlSAGUE, X.; XAVIER, F.; BOSCH, M. D.; MUÑOZ, M. D. The male role in cervical cancer. Salud Pública de México, Cuernavaca, v. 45, p. 345353, 2003. 
CASTRO, T. M. P. P. G.; BUSSOLOTI FILHO, I.; NASCIMENTO, V. X.; XAVIER, S. D. Detecção de HPV na mucosa oral e genital pela técnica PCR em mulheres com diagnóstico histopatológico para HPV genital. Revista Brasileira de Otorrinolaringolia, São Paulo, v.75, p.167-171, 2009.

CHATURVEDI, A. K. Beyond cervical cancer: burden of other HPV related cancers among men and women. Journal Adolescent Health, Rockville, v. 46 , p. $20-26,2010$.

CONTI, F. S.; BORTOLIN, S.; KULKAMP, C. I. Educação e promoção à saúde: comportamento e conhecimento de adolescentes de colégio público e particular em relação ao papilomavírus humano. Jornal Brasileiro de Doenças Sexualmente Transmissíveis, Rio de Janeiro, v. 18, p. 30-35, 2006.

EINSTEIN M. H.; SCHILLER, R. P. STRICKER, H. D.; COURSAGET, P; JEKINS, D. Clinician's guide to human Papillomavirus immunology: knowns and unknowns. The Lancet Infectious Diseases, Washington, v. 9, p. 347-56, 2009.

FEHRMANN, F.; LAIMINS, L. A. Human papillomaviruses: targeting differentiating epithelial cells for malignant transformation. Oncogene, New York, v. 22, p. 5201-5207, 2003.

FELLER, L.; KHAMMISSA, R. A.; WOOD, N. H. Epithelial maturation and molecular biology of oral HPV. Infectious Agents and Cancer, Washington, v. 4, p. 16, 2009.

FERNANDES, A. M. S.; GASIANI, A. D.; BAHAMONDES, L. G.; CUPERTINO, C. V. Conhecimento, atitudes e práticas de mulheres brasileiras atendidas pela rede básica de saúde com relação às doenças de transmissão sexual. Cadernos de Saúde Pública, Rio de Janeiro, v. 16, p. 103-112, 2000.

FIFE, K. H.; KATZ, B. P.; ROUSH, J.; HANDY, V. D.; BROWN, D. R.; HANSELL, R. Cancer associated human papillomavirus: types are selectively increased in the cervix of women in the first trimester of pregnancy. American Journal of Obstetrics and Gynecology, Washington, v. 174, p. 1487-1493, 1996.
FRIGATO, S.; HOGA, A. K. Assistência à mulher com câncer de colo uterino: o papel da enfermagem. Revista Brasileira de Cancerologia, Rio de Janeiro, v. 49, n. 4, p. 209-214, 2003.

HARPER, D. M.; FRANCO, E. L., WHEELER, C. M., MOSCICKI, A. B., ROMANOWSKI, B., ROTELI-MARTINS, C. M.; DUBIN, G. Sustained virus-like particle vaccine against human Papillomavirus types 16 and 18: followup from a randomized control trial. The Lancet, Nars City, v. 367, p. 1247-1255, 2006.

KAHN, J. A.; ROSENTHAL, S. L.; SUCCOP, P. A.; HO, G. Y.; BURK, R. D. The interval 12. between menarche and age of first sexual intercourse as a risk factor for subsequent HPV infection in adolescent and young adult women. The Journal of Pediatrics, Cincinnati, v. 141, p. 718-723, 2002a.

KAHN, J. A.; ROSENTHAL, S. L.; SUCCOP, P. A.; HO, G. Y.; BURK, R. D . Mediators of the 16. Association between age of first sexual intercourse and subsequent human papillomavirus infection. The Journal of Pediatrics, Cincinnati, v.109, p. e5-e5, 2002 b.

LINHARES, A. C.; VILLA L. L. Vaccines against rotavirus and human papillomavirus (HPV). The Journal of Pediatrics, Cincinnati, v. 82, n. 3, p. 25-34, 2006.

LONGWORTH, M. S., LAIMINS, L. A. Pathogenesis of human papillomaviruses in differentiating epithelia. Microbiology and Molecular Biology Reviews, Washington, v. 68, p. 362-372, 2004.

MADUREIRA, V. S. F.; TRENTINI, M. Da utilização do preservativo masculino à prevenção de DST/aids. Ciência \& Saúde Coletiva, Rio de Janeiro, v. 13, p. 1807-1816, 2008.

MARTINS FILHO, P. R. S.; PIVA, M. R.; SANTOS, T. S.; ANDRADE, E. S. D. S.; SILVA, L. C. F. D. Papiloma de células escamosas da cavidade oral. Revista de Cirurgia e Traumatologia Buco-maxilo-facial, Camaragibe, v. 9, p. 69-78, 2009. 
MURTA, E. F. C.; SOUZA, M. A. H.; JÚNIOR, E. A.; ADAD, S. J. Aspectos epidemiológicos da infecção pelo papilomavírus humano. Jornal Brasileiro de Ginecologia e Obstetrícia, Rio de Janeiro, v. 107, p. 95-99, 1997.

MURTA, E. F. C.; SOUZA, M. A. H.; ARAÚJO JÚNIOR, E.; ADAD, S. J. Infecção pelo papilomavirus humano em adolescentes: relação com o método anticoncepcional, gravidez, fumo e achados citológicos. Revista Brasileira de Ginecologia e Obstetrícia, Rio de Janeiro, v. 23, p. 217-221, 2001.

NASCIMENTO, M. V.; SOUZA, I.; DEUS, M. S. M.; PERON, A. O que sabem os adolescentes do ensino básico público sobre o HPV. Semina: Ciências Biológicas e da Saúde, Londrina, v. 34, n. 2, p. 229-238, 2013.

PINHO, A. A.; FRANÇA-JUNIOR, I. Prevenção de câncer de colo de útero: um modelo teórico para analisar o acesso e a utilização do teste de Papanicolaou. Revista Brasileira de Saúde Materno Infantil, Recife, v. 3, n. 1, p. 95-112, 2003.

PINTO, A P.; TULIO, S.; CRUZ, O. R. Cofatores do HPV na oncogênese cervical. Revista da Associação Médica Brasileira, São Paulo, v. 48, p. 73-78, 2002.

PICOS. Prefeitura Municipal. História e potencialidades. 2008. Disponível em: <http:// www.picos.pi.gov.br/conhecapicos.asp $>$. Acesso em: 6 abr. 2013.

QUEIROZ, D. T.; PESSOA, S. M. F.; SOUSA, R. A. D. Infecção pelo papiloma vírus humano (HPV): incertezas e desafios. Acta Paulista de Enfermagem, São Paulo, v. 18, p. 190-196, 2005.

RAMOS, S.P. HPV - Papiloma Vírus um novo capítulo nas infecções vaginais. 2011. Disponível em: $\quad<$ http://www.gineco.com.br/hpvum.htm $>$. Acesso em: 15 jul. 2013.

REIS, A. A.; BARCELOS, L.; DE PAULA, A. A. P.; DA CRUZ, A. D. Aspectos clínicos epidemiológicos do câncer de pênis. Revista de Ciência \& Saúde Coletiva, v.15, p. 1105-1111, 2010.
SAITO, M. I. Adolescência e contracepção de emergência. Revista Paulista de Pediatria, São Paulo, v. 25, p. 180-186, 2007.

SANTOS, S. H. R.; ZEFERINO, L.; VILLA, L. L.; SOBRINHO, J. P.; AMARAL, R. G.; MAGALHÃES, A. V. D. Human Papillomavirus prevalence among women with cervical intraepithelial neoplasia III and invasive cervical cancer from Goiânia, Brazil. Memórias do Instituto Oswaldo Cruz, Rio de Janeiro, v. 98, p. 181-184, 2003.

SCHIFFMAN, M.H.; BAUER, H. M.; HOOVER, R. N.; GLASS, A. G.; CADELL, D. M.; RUSH, B. B.; MANOS, M. M. Epidemiologic evidence showing that human papillomavirus infection causes most cervical intraepithelial neoplasia. Journal of the National Cancer Institute, Bethesda, v. 85, p. 958-64, 1993.

SCHUSSEL, E. Y. O sexo na adolescência. São Paulo: Atheneu, 2004.

SCULLY, C. Oral squamous cell carcinoma; from an hypothesis about a virus, to concern about possible sexual transmission. Oral Oncology, Phyladelphia, v. 38, p. 227-234, 2002 .

SILVA, A. J.; CRUZ, V. S. Conhecimento do HPV entre os adolescentes de 14 a 17 anos. 2010. Disponível em: $<$ http://www.webartigos. com/artigos/conhecimento-do-hpv-entre-osadolescentes-de-14-a-17-anos/34000/> Acesso em: 25 fev. 2013.

SILVA, E. D. C.; MEDEIROS, R. C. D.; MORAES, L. M. D.; MENEZES FILHO, J. B. D.; RAMOS, E. S. N.; SATURNINO, A. C. R. D. Papilomavirus humano. Revista Brasileira de Análises Clínicas, São Paulo, v. 36, p. $137-$ 142, 2004.

SILVEIRA, G. A. S.; FERRAZ, B. G.; CONRADO, G. A. M. Conhecimento dos universitários sobre HPV e câncer de colo uterino em uma faculdade privada localizada no sertão de Pernambuco. Saúde Coletiva em Debate, Manguinhos, v. 2, p. 87-95, 2012. 
SMITH, J. S.; MELENDY, A.; RANA, R. K.; PIMENTA, J. M. Age-specific prevalence of infection with human papillomavirus in females: a global review. Journal Adolescents Health, Philadelphia, v. 43, p. 5-25, 2008.

TAQUETTE, S. R.; VILHENA, M. M.; PAULA, M. C. Doenças sexualmente transmissíveis na adolescência: estudo de fatores de risco. Medicina Tropical, São Paulo, v. 37, p. 210214, 2004.

VILLA, L. L.; COSTA, R. L.; PETTA, C. A.; ANDRADE, R. P.; AULT, K. A.; GIULIANO, A. R. Prophylactic quadrivalent human papillomavirus (types 6, 11, 16, and 18) L1 virus-like particle vaccine in young women: a randomized double-blind placebo-controlled multicentre phase II efficacy trial. Lancet Oncology, Washington, v. 6, p. 271-8, 2005.

WORLD HEALTH ORGANIZATION (WHO). Human papillomavirus (HPV). Disponível em:<http://www.who.int/immunization/topics/ hpv/en/>. Acesso em: 14 mar. 2013.

YOST, N. P.; SANTOSO, J. T.; MCINTIRE, D. D.; ILIYA, F. A. Postpartum regression rates of antepartum cervical intraepithelial Neoplasia II and III lesions. Obstetrics and Ginecology, Nars City, v. 93, p. 359-362, 1999. 
